Original article

\title{
Psychiatric disorders and autism in young children with 22q11.2 deletion syndrome compared to children with idiopathic autism
}

\author{
Yaffa Serur ${ }^{\mathrm{a}}$, Dafna Sofrin Frumer ${ }^{\mathrm{b}}$, Keren Daon ${ }^{\mathrm{b}}$, Dolly Sobol-Havia ${ }^{\mathrm{b}}$, \\ Ronnie Weinberger ${ }^{\mathrm{c}}$, Cory Shulman ${ }^{\mathrm{d}}$, Doron Gothelf ${ }^{\mathrm{b}, \mathrm{c}, *}$ \\ a Beer-Sheva Mental Health Center, Hazadik Miyerushalaim 2, Beer Sheva 8461144, Israel \\ ${ }^{\mathrm{b}}$ The Child Psychiatry Division, The Edmond and Lily Safra Children's Hospital, Sheba Medical Center, Tel Hashomer, 5262000, Israel \\ ' Sackler Faculty of Medicine and Sagol School of Neuroscience, Tel Aviv University, Tel Aviv 69978, Israel \\ ${ }^{\mathrm{d}}$ The Paul Baerwald School of Social Work and Social Welfare, Hebrew University of Jerusalem, Israel
}

\section{A R T I C L E I N F O}

\section{Article history:}

Received 16 July 2018

Received in revised form 24 October 2018

Accepted 25 October 2018

Available online 16 November 2018

\section{Keywords:}

22q11.2 Deletion syndrome

Idiopathic autism

ASD

Preschool

Psychiatric disorders

\begin{abstract}
A B S T R A C T
Background: The 22q11.2 deletion syndrome (22q11DS) is a neurogenetic condition characterized by high rates of psychiatric disorders. To our knowledge, this is the first study to assess psychiatric disorders in young children with 22q11DS using a structured psychiatric diagnostic interview, and one of few studies to use the complete gold standard diagnostic evaluation to examine the prevalence of autism spectrum disorder (ASD) in young children with 22q11DS and compare it to a matched control group with iASD.

Methods: We identified the psychiatric disorders and autistic phenotype of young children with 22q11DS (age 3-8 years) and compared them with those of age and sex-matched children with idiopathic autism (iASD). We used the gold standard psychiatric and ASD assessments including the Autism Diagnostic Interview-Revised (ADI-R), the Autism Diagnostic Observation Schedule (ADOS) and a clinical examination by a child psychiatrist.

Results: Eighty-four percent of the children with 22q11DS had at least one psychiatric disorder, including anxiety disorders and ADHD, and 16\% met strict criteria for ASD. Children with 22q11DS and ASD symptoms had less severe overall ASD symptoms than those with iASD. Children with 22q11DS, regardless of ASD diagnosis, were characterized by repetitive restricted behaviors.

Conclusions: Our results highlight the need to screen for psychiatric disorders in 22q11DS and treat them already in preschool years.
\end{abstract}

(c) 2018 Elsevier Masson SAS. All rights reserved.

\section{Introduction}

The 22q11.2 deletion syndrome (22q11DS) is a neurogenetic condition caused by hemizygous microdeletion of the long arm of chromosome 22 . Approximately $90 \%$ of individuals with 22q11DS carry a $3 \mathrm{Mb}$ deletion, and the rest carry smaller $(1.5 \mathrm{Mb})$ and atypical deletions [1]. The syndrome occurs in 1-2,000-4000 live births and is characterized by medical comorbidities (e.g., cardiovascular and cleft anomalies) and high rates of psychiatric disorders, developmental delays, and average IQ within the borderline range [2-4].

Most studies to date have assessed the psychiatric phenotype of older children, adolescents and adults with 22q11DS (see

\footnotetext{
* Corresponding author at: The Behavioral Neurogenetics Center, The Edmond and Lily Safra Children's Hospital, Sheba Medical Center, Tel Hashomer, 5262000, Israel.

E-mail address: gothelf@post.tau.ac.il (D. Gothelf).
}

Schneider et al., 2014 for review [5]). Those studies found that $\sim 70 \%$ individuals with 22q11DS have at least one psychiatric disorder, including schizophrenia spectrum disorders evolving by early adulthood in about one-third of the patients, and high rates of attention deficit/hyperactivity disorder (ADHD), anxiety disorders, mood disorders and autism spectrum disorder (ASD) [5,6].

There are, however, very few studies that assessed the severity of psychiatric symptoms in preschool and young children with 22q11DS, and none before the age of 6 years [6]. Two studies that examined behavior and cognition in preschool children with 22q11DS [7,8] reported psychiatric problems based on the child behavior checklist (CBCL), a parent-report questionnaire, and were not based on structured psychiatric diagnostic interviews, such as the Schedule for Affective Disorders and Schizophrenia (K-SADS), which is designed for diagnosing the presence or absence of psychiatric disorders. Based on the CBCL, children with 22q11DS had high mean scores on the CBCL scales of withdrawn behavior, attention, affective, and pervasive developmental problems [7,8]. 
While the rates of psychiatric disorders in young children with 22q11DS are unknown, the rates of ASD in individuals with 22q11DS are a matter of controversy. Several studies assessed the presence of ASD in individuals with 22q11DS and reported rates of ASD that varied greatly, i.e., from $7 \%$ to 50\% [6,9-15]. Possible explanations for such variability include differences in the age of the subjects and in the tools used to assess ASD. Of note, most studies on 22q11DS relied on interviews with the parents using the Autism Diagnostic Interview-Revised (ADI-R) $[6,10,11,15]$ while only a few of them used the Autism Diagnostic Observation Schedule (ADOS) [9,14]. The ADOS relies on direct observation of the child's interaction abilities and behavior by a qualified examiner and is one of the gold-standard diagnostic instruments for the diagnosis of autism.

In addition to using gold standard measure tools, to better understand the autism phenotype in 22q11DS it is important to compare the social and repetitive symptoms observed in children with 22q11DS to those of children with idiopathic autism (iASD). In the only study to date that compared ASD symptoms exhibited by 22q11DS children aged 6-15 years with age- and sex-matched children with iASD [11], the findings of the ADI-R revealed that both groups were equally affected in reciprocal social interaction and stereotyped behavior domains, while those with 22q11DS were less affected in the communication domains.

The overall aim of the present study was to identify the psychiatric disorders and rates and characteristics of autism in young children with 22q11DS, and to compare them to age and sex-matched young children with iASD. Specifically, we evaluated the prevalence of psychiatric disorders and ASD in young children with 22q11DS using comprehensive gold standard measures and compared them to the results of those assessments that were obtained from an age and sex-matched group of children with iASD. Lastly, we wished to compare the severity of psychiatric symptoms and the autistic phenotype of 22q11DS children diagnosed with autism compared to 22q11DS children without autism.

The study hypotheses were: 1 . Children with 22q11DS will have lower percentages of ASD diagnosis than reported in past studies, which relied solely on parents' interviews. 2. We expect to find high rates of psychiatric disorders in 22q11DS, especially ADHD and anxiety disorders. 3. Children with 22q11DS will show similar high scores to children with iASD on a behavioral checklist. 4 . The social phenotype of 22q11DS children will be overall less impaired than that of iASD in most domains, except repetitive and restricted behaviors, which will be equally high in both populations.

\section{Methods}

\subsection{Participants}

Twenty-five children (18 males, 7 females, aged 3-8 years [mean \pm SD $5.57 \pm 1.55$ years]) with a genetically confirmed diagnosis of 22q11DS using fluorescent in situ hybridization or chromosomal micro-array tests, were recruited from our Behavioral Neurogenetics Center at Sheba Medical Center. The Center coordinates research and comprehensive medical and psychiatric treatments of individuals with 22q11DS throughout the country, and children with 22q11DS are referred to the Center from genetic departments and parents' associations nationwide. Note that our sample was not biased towards more affected children as none of the children recruited was referred for evaluation of suspected autism.

The control group consisted of 28 children with iASD who were recruited from the Preschool Day Care Program for Children with ASD at the same medical center. These children are referred to the program through the local municipality and represent preschoolers from the community with a range of ASD severity. The ASD group was evaluated by a clinical geneticist and developmental pediatricians, and their medical records were reviewed. Those children were referred for genetic testing when there was any suspicion of a genetic syndrome and were excluded from the study if that suspicion was confirmed. The matching procedure began with the ASD sample, which consisted of a 3.6:1 male to female ratio. The demographic characteristics of the study and control groups are presented in Table 1. The Sheba Medical Center Review Board approved the protocol of the study design, and informed consent was provided by the parents of all participants.

\subsection{Measures}

\subsubsection{Psychiatric evaluations}

The parents of all the study participants completed the $\mathrm{CBCL}$ preschool and school versions [16,17]. In addition, the parents of the children with 22q11DS were interviewed by a child psychiatrist using the semi-structured K-SADS to screen for the presence of DSM-5 psychiatric disorders in their children. To reliably assess the psychiatric diagnoses in our preschool sample, we adapted the KSADS examinations to preschool children, as has done previously in studies that validated the use of the K-SADS in non-22q11DS preschool children [18].

\subsubsection{Evaluation of ASD symptoms}

All participants underwent the gold standard assessments of ASD [19] including the ADI-R [20] and the Autism Diagnostic Observation Schedule, second edition (ADOS-2) [21]. They also underwent a clinical examination by a child psychiatrist in order to confirm the diagnosis and severity of autism. The ADI-R interview generates scores in three areas of content (communication and language, social interaction, and restricted, repetitive behaviors) and is based on DSM-IV and ICD-10 criteria for autism. A classification of autism was assigned when scores in all three areas met or exceeded the specified cutoffs. In line with the analysis of Bishop et al. [22] we used the nonverbal algorithm scores of the ADI-R to compare between groups because some of the children in our sample were not verbal.

The ADOS-2 [23] is a standardized observational assessment that consists of various activities aimed to elicit behaviors for evidencing the quality of social interactions and communicative abilities, as well as repetitive and restrictive behaviors associated

Table 1

Demographic characteristics of the study sample.

\begin{tabular}{llll}
\hline & $\begin{array}{l}22 \mathrm{q} 11 \mathrm{DS} \\
(\mathrm{n}=25)\end{array}$ & $\begin{array}{l}\text { iASD } \\
(\mathrm{n}=28)\end{array}$ & Statistics \\
\hline Sex (M/F) & $18 / 6$ & $22 / 6$ & $\chi^{2}(0.30), p=0.58$ \\
Age, years & $5.57(1.55)$ & $4.94(1.30)$ & $\mathrm{t}(1.51)=1.60, \mathrm{p}=0.12$ \\
Age range, years & $3-8$ & $3-8$ & \\
Full-scale IQ (SD) & $87.72(16.05)$ & $74.29(24.28)$ & $\mathrm{t}(1.51)=2.39, \mathrm{p}=0.02$ \\
Mother's education & & & $\chi^{2}=3.67, \mathrm{p}=0.30$ \\
Elementary school & $0(0 \%)$ & $1(3.6 \%)$ & \\
High school & $8(34.8 \%)$ & $4(14.3 \%)$ & \\
Vocational & $2(8.7 \%)$ & $4(14.3 \%)$ & \\
Academic degree & $13(56.5 \%)$ & $19(67.9 \%)$ & \\
ADOS-2 modules & & & \\
1 & $3(12.0 \%)$ & $7(25.0 \%)$ & \\
2 & $11(44.0 \%)$ & $8(28.6 \%)$ & \\
3 & $11(44.0 \%)$ & $13(46.4 \%)$ & \\
Intelligence measures & & & \\
WPSSI-III & $4(16.0 \%)$ & $9(32.1 \%)$ & \\
Short WPPSI & $15(60.0 \%)$ & $1(3.6 \%)$ & \\
Mullen & $1(4.0 \%)$ & $16(57.1 \%)$ & \\
Short WISC-IV & $4(16.0 \%)$ & $2(7.1 \%)$ & \\
Bayley-IV & $1(4.0 \%)$ & $0(0 \%)$ & \\
\hline
\end{tabular}

Abbreviations: 22q11DS: 22q11 deletion syndrome; iASD: idiopathic autism; ADOS-2: autism diagnostic observational schedule; WPSSI: wechsler preschool and primary scale of intelligence; WISC: wechsler intelligence scale for children. 
with autism. We used three modules of the ADOS-2 in the current study: module 1 for participants with no speech and/or singleword vocabularies, module 2 for participants with phrase speech, and module 3 for participants with fluent speech (see Table 1 for the distribution of the modules). In addition to the ASD and autism cutoff scores, we used the diagnostic algorithms and calibrated scores for the overall severity score [24], as well as the calibrated severity scores for social affect and restricted and repetitive behaviors [25]. These calibrated scores were standardized to allow comparison of symptom domains across modules of the ADOS- 2 .

\subsubsection{Cognitive assessment}

The general cognitive level was measured by the short version (vocabulary and block design tasks) [26] or the full version of the Wechsler Preschool and Primary Scale of Intelligence (WPPSI-III) for children aged 2-7 $1 / 2$ years [27], or the Wechsler Intelligence Scale for children version IV (WISC-IV) for children older than $7^{1 / 2}$ years [28]. Some of the young children were assessed using the Mullen Scales of Early Learning [29,30, see Table 1 for the distribution of the Wechsler versions].

\subsubsection{Data analysis}

Data were analyzed using SPSS software for Mac Version 25. Categorical variables were compared by Pearson's $\chi 2$ tests for proportions analysis. Continuous variables were compared by independent sample t-tests. Comparisons between the 22q11DS children with ASD to iASD and between 22q11DS with vs. without ASD were made using the Mann Whitney test.

\section{Results}

\subsection{Rates of psychiatric disorders in 22q11DS}

Of the 22q11DS group, 84\% met DSM-5 diagnostic criteria for at least one psychiatric disorder, and the mean number of psychiatric diagnoses in that group was $2.04 \pm 1.51$. The prevalence of 22q11DS psychiatric diagnoses was: $\mathrm{ADHD}=60 \%$, elimination disorders $=$ $52 \%$, anxiety disorders $=36 \%$ and oppositional defiant disorder $=$ $20 \%$. None of the children fulfilled the criteria for a psychotic disorder (see Table 2).

\subsection{Rates of $A S D$ in $22 q 11 D S$}

Based on the ADOS-2, the ADI-R and best clinical judgment, only four children with 22q11DS (16\%) fulfilled the DSM-5 criteria for ASD. Four children (16\%) were diagnosed as having autism on the ADI-R, and 12 children (48\%) were diagnosed as being positive for any of the

Table 2

Psychiatric disorders in children with 22q11DS.

\begin{tabular}{ll}
\hline Psychiatric disorders & $\mathrm{n}(\%)$ \\
\hline Any psychiatric disorder in 22q11DS & $21(84)$ \\
Any psychiatric disorder w/o elimination disorders & $18(72)$ \\
Any anxiety disorder & $9(36)$ \\
Specific phobia & $7(28)$ \\
Social anxiety disorder & $1(4)$ \\
Separation anxiety disorder & $4(16)$ \\
Generalized anxiety disorder & $2(8)$ \\
Elimination disorder & $13(52)$ \\
Enuresis & $11(44)$ \\
Encopresis & $5(20)$ \\
ADHD & $15(60)$ \\
ADHD combined presentation & $8(32)$ \\
ADHD inattentive presentation & $6(24)$ \\
ADHD hyperactive presentation & $1(4)$ \\
Oppositional defiant disorder & $5(20)$ \\
\hline
\end{tabular}

Abbreviations: 22q11DS: 22q11 deletion syndrome; ADHD: attention deficit/ hyperactivity disorder.
ADI-R domains. Within the different ADI-R domains, ten children (40\%) were above the cutoff score of reciprocal social interaction, eight children (32\%) above the communication cutoff score, and 12 children (48\%) above the restricted, repetitive and stereotyped behavior cutoff score. Five children (20\%) fulfilled the diagnostic criteria for ASD on the ADOS-2. One child that fulfilled the ADOS-2 but not the ADI-R criteria for ASD was judged clinically not to have ASD, but did fulfill the DSM-5 criteria for generalized anxiety disorder.

\subsection{Comparison of autistic and psychiatric symptoms severity}

\subsubsection{2q11DS versus $i A S D$}

Children with idiopathic autism had significantly higher overall and subscale scores on the ADOS- 2 and the ADI-R compared to children with 22q11DS ( $p<0.050$ for all, see Table 3 ). Severity of psychiatric symptoms, as measured by the CBCL, was similar for the children with 22q11DS and those with iASD for all domains except for the scores for withdrawal, which were significantly higher in the iASD group ( $p=0.008$, Table 3$)$.

\subsubsection{2q11DS with ASD versus iASD}

The overall calibrated scores of the ADOS-2 were significantly higher in children with iASD compared to 22q11DS with ASD ( $p=$ 0.007 Mann Whitney). Within the ADOS-2 subscales, the social affect calibrated scores were higher in the iASD group than in the 22q11DS with ASD group ( $p=0.002$ ), while the repetitive restricted behavior calibrated scores did not differ significantly between groups $(p=0.094)$. Comparison of the specific items within the ADOS-2 revealed that children with 22q11DS and ASD were less impaired than children with iASD in nonverbal communication items, including gestures $(p=0.028)$ and facial expressions $(p=$ 0.017). Within the ADI-R domains, there were no significant differences between 22q11DS with ASD and the iASD group.

There were no significant differences in the severity of psychiatric symptoms measured by the CBCL for the children with 22q11DS with ASD and those with idiopathic autism for all domains (see Table 3).

\subsubsection{2q11DS with ASD versus 22q11DS without $A S D$}

Children with 22q11DS with ASD scored significantly higher in the overall calibrated scores of the ADOS-2 compared to 22q11DS without ASD $(p<0.001)$. Within the ADOS-2 subscales, the social affect calibrated scores were also higher in the 22q11DS with ASD group than in the 22q11DS without ASD $(p=0.001)$. Conversely, the repetitive restricted behavior calibrated scores did not differ significantly between groups $(p=0.068)$. When comparing the severity of psychiatric symptoms on the $\mathrm{CBCL}$, there were no significant differences between groups for all domains. There were also no differences in cognitive abilities between 22q11DS groups as indicated by FSIQ scores (see Table 3).

\section{Discussion}

To our knowledge, this is the first study to assess psychiatric disorders in young children with 22q11DS using the approach of a structured psychiatric diagnostic interview. It is also one of a few studies to use the complete gold standard diagnostic evaluation (i.e., ADI-R + ADOS + clinical evaluation) to examine the prevalence of ASD in young children with 22q11DS and to compare it to a matched control group of children with iASD.

\subsection{Rates of ASD in 22q11DS}

In accordance with our hypothesis, young children with 22q11DS showed lower rates of ASD diagnosis (16\%) using the 
Table 3

Comparison of severity of autistic and psychiatric symptoms among study groups.

\begin{tabular}{|c|c|c|c|c|c|c|c|}
\hline & $\begin{array}{l}22 q 11 D S \\
\text { total }\end{array}$ & $\begin{array}{l}\text { 22q11DS with ASD } \\
(\mathrm{n}=4)\end{array}$ & $\begin{array}{l}\text { 22q11DS without ASD }(\mathrm{n}= \\
\text { 21) }\end{array}$ & $\begin{array}{l}\text { iASD } \\
(n=28)\end{array}$ & $\begin{array}{l}\text { 22q11DS v.s } \\
\text { iASD }\end{array}$ & $\begin{array}{l}\text { 22q11DS+ } \\
\text { ASD v.s } \\
\text { iASD }\end{array}$ & $\begin{array}{l}\text { 22q11DS+ASD v. } \\
\mathrm{s} \\
\text { 22q11DS-ASD }\end{array}$ \\
\hline ADOS-2 & & & & & $\mathrm{t}(1.51)$ & & \\
\hline Overall & $2.28(1.49)$ & $4.75(1.50)$ & $1.81(0.93)$ & $7.79(1.73)$ & $\mathrm{p}<0.001$ & $\mathrm{p}=0.007$ & $\mathrm{p}<0.001$ \\
\hline Social affect & $2.64(1.70)$ & $5.25(1.26)$ & $2.14(1.28)$ & $8.11(1.40)$ & $\mathrm{p}<0.001$ & $\mathrm{p}=0.002$ & $\mathrm{p}=0.001$ \\
\hline $\begin{array}{l}\text { Restricted and repetitive behavior } \\
\text { ADI-R }\end{array}$ & $3.40(2.26)$ & $5.50(1.91)$ & $3.00(2.12)$ & $7.39(2.17)$ & $\mathrm{p}<0.001$ & $\mathrm{p}=0.094$ & $p=0.068$ \\
\hline Reciprocal social & $8.40(6.33)$ & $16.00(5.22)$ & $6.95(5.50)$ & $\begin{array}{l}18.86 \\
(6.01)\end{array}$ & $\begin{array}{l}\mathrm{t}(1.51), \mathrm{p}< \\
0.001\end{array}$ & $\mathrm{p}=0.361$ & $\mathrm{p}=0.011$ \\
\hline Communication (non verbal) & $4.84(4.69)$ & $11.25(2.21)$ & $3.62(3.98)$ & $8.52(4.57)$ & $\begin{array}{l}\mathrm{t}(1.50), \mathrm{p}= \\
0.006\end{array}$ & $\mathrm{p}=0.376$ & $p=0.006$ \\
\hline $\begin{array}{l}\text { Restricted, repetitive and stereotyped } \\
\text { behavior }\end{array}$ & $2.00(2.41)$ & $5.00(2.94)$ & $1.43(1.89)$ & $6.61(2.96)$ & $\begin{array}{l}\mathrm{t}(1.51), \mathrm{p}< \\
0.001\end{array}$ & $\mathrm{p}=0.424$ & $\mathrm{p}=0.006$ \\
\hline FSIQ & $\begin{array}{l}87.72 \\
(16.05)\end{array}$ & $85.25(14.08)$ & $88.19(16.67)$ & $\begin{array}{l}74.29 \\
(24.28)\end{array}$ & $\mathrm{t}(1.51), \mathrm{p}=0.02$ & $\mathrm{p}=0.305$ & $\mathrm{p}=0.592$ \\
\hline CBCL & & & & & $\mathrm{t}(1.50)$ & & \\
\hline Anxious/depressed & $\begin{array}{l}57.58 \\
(10.22)\end{array}$ & $67.50(20.34)$ & $55.60(6.04)$ & $\begin{array}{l}55.25 \\
(6.54)\end{array}$ & $\mathrm{p}=0.325$ & $\mathrm{p}=0.457$ & $\mathrm{p}=0.431$ \\
\hline Somatic complaints & $57.13(7.56)$ & $57.75(8.38)$ & $57.00(7.62)$ & $\begin{array}{l}56.04 \\
(6.31)\end{array}$ & $\mathrm{p}=0.574$ & $\mathrm{p}=0.527$ & $\mathrm{p}=0.852$ \\
\hline Withdrawn & $58.33(9.40)$ & $70.25(16.82)$ & $55.95(5.14)$ & $\begin{array}{l}65.86 \\
(9.96)\end{array}$ & $\mathrm{p}=0.008$ & $\mathrm{p}=0.564$ & $\mathrm{p}=0.081$ \\
\hline Attention problems & $59.50(9.61)$ & $63.25(14.22)$ & $58.75(8.73)$ & $\begin{array}{l}57.68 \\
(6.83)\end{array}$ & $\mathrm{p}=0.430$ & $p=0.602$ & $\mathrm{p}=0.627$ \\
\hline Aggressive behavior & $55.42(6.33)$ & $58.50(8.43)$ & $54.80(5.91)$ & $\begin{array}{l}56.00 \\
(8.35)\end{array}$ & $\mathrm{p}=0.781$ & $\mathrm{p}=0.491$ & $\mathrm{p}=0.388$ \\
\hline Internalizing & $\begin{array}{l}54.25 \\
(13.10)\end{array}$ & $62.50(19.43)$ & $52.60(11.44)$ & $\begin{array}{l}59.71 \\
(9.47)\end{array}$ & $\mathrm{p}=0.088$ & $\mathrm{p}=0.527$ & $\mathrm{p}=0.273$ \\
\hline Externalizing & $52.79(9.64)$ & $56.75(11.59)$ & $52.00(9.35)$ & $\begin{array}{l}54.61 \\
(9.03)\end{array}$ & $\mathrm{p}=0.487$ & $\mathrm{p}=0.640$ & $p=0.477$ \\
\hline Total problem & $\begin{array}{l}51.75 \\
(12.38)\end{array}$ & $56.50(17.71)$ & $50.80(11.42)$ & $\begin{array}{l}51.82 \\
(8.58)\end{array}$ & $\mathrm{p}=0.981$ & $\mathrm{p}=0.602$ & $\mathrm{p}=0.388$ \\
\hline
\end{tabular}

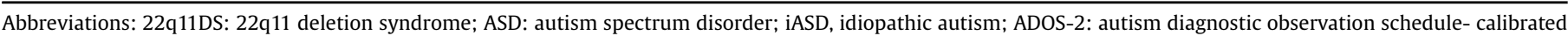
scores; ADI-R: autism diagnostic interview; FSIQ: full scale IQ; CBCL: child behavior checklist.

comprehensive gold standard diagnostic evaluation for ASD that included ADOS evaluations of the children in contrast to most previous studies in 22q11DS that relied solely on interviews with the parents as a single diagnostic tool $[6,10,11,15]$.

The $16 \%$ rate of ASD found in our study is in line with the $14 \%$ rate reported in a study by Fine et al. [10] on younger children (2-12 years) with 22q11DS. Of note, most studies that reported higher rates of ASD in individuals with 22q11DS (20\%-50\%) were conducted on older children and adults $[6,11,12,13,15]$. Three of the four children in our study with 22q11DS who were diagnosed with ASD met the criteria for ASD on the ADOS-2, but did not meet the ADOS-2 criteria for Autism, in line with previous studies identifying that a less severe form of ASD is common in 22q11DS [12,13].

As previously mentioned, one potential explanation for the lower rate of ASD in our study compared to some previous studies $[6,15]$ could be related to differences in methods of assessment of autism between our research and others. Previous studies on ASD and 22q11DS that relied only on parents' interviews using the ADI$\mathrm{R}[6,11,15]$ reported higher ASD rates in 22q11DS (20-50\%), while the studies that used an observational diagnostic instrument like the ADOS [9,14] reported lower rates of ASD (0-18\%), suggesting that adding observational measures of ASD to parent-report measures provide lower rates of ASD diagnosis in 22q11DS.

Another potential explanation for the relatively low ASD rates is the high rates of ADHD found in our young sample, which may suggest that some individuals diagnosed with ADHD could switch diagnosis to ASD later during childhood and adolescence. This has been reported in non-22q11DS preschool children with ADHD: about one-third of those with remitted ADHD were diagnosed with ASD at age 12 years [31]. Longitudinal studies beginning in preschool years and focused on ADHD and ASD in 22q11DS are needed to verify our assumption that some young children with ADHD might switch to ASD later in childhood and adolescence.
A third potential explanation for the low ASD rates found may be that children with high comorbid psychiatric or neurologic disorders, like in 22q11DS, were found to be diagnosed with ASD later than children without such comorbidities [32]. This example, along with the diagnostic overlap between ADHD and ASD, raises the possibility that ADHD and neurological conditions may overshadow the diagnosis of ASD in 22q11DS. It is important to note that since there is a high proportion of males in our 22q11DS sample, the rates of ASD found in our study are most likely to be higher than if our 22q11DS sample was balanced in terms of the sex distribution.

\subsection{Psychiatric diagnoses in 22q11DS}

As hypothesized, we found high rates of psychiatric comorbidities in our cohort of young 22q11DS children, including ADHD (60\%) and anxiety disorders (36\%).

Although a high prevalence of ADHD and anxiety disorders has been reported in previous 22q11DS studies [5], none of them assessed children under the age of six years [5], and none examined young children with 22q11DS using a structured psychiatric diagnostic interview.

The diagnosis of psychiatric disorders in preschool children with developmental disorders can be a complex endeavor and can probably explain why this is the first study that conducted a structured psychiatric evaluation of 22q11DS preschool children. Psychiatric disorders can be reliably diagnosed already in preschool age. For example, the Diagnostic Classification of Mental Health and Developmental Disorders of infancy and Early Childhood (DC:0-5) points out that ADHD can be diagnosed from age 36 months and provides the same specifiers- inattentive and hyperactive-impulsive - similarly to the ADHD specifiers in older children [33]. 
While the $36 \%$ rate of anxiety disorders found in our sample of young children with 22q11DS is similar to that reported in older children and adolescents with 22q11DS [5], the 60\% rate of ADHD in our sample was much higher than the $37 \%$ that had been reported in previous studies on school-age children with 22q11DS [5]. A recent longitudinal study demonstrated that the rate of ADHD in 22q11DS declines from $37 \%$ in children age $6-12$ years to $16 \%$ in adults [5]. Taken together with the findings from our study, it seems that there is a decline in ADHD rates from preschool years to childhood to adulthood. The higher rates of ADHD in preschoolers with 22q11DS is also in line with findings from longitudinal studies of non-22q11DS children showing that $30 \%$ of preschoolers diagnosed with ADHD at the age 5 no longer met ADHD criteria at age 12, and several of them are diagnosed instead with different psychiatric disorders including anxiety disorders, ASD and learning disorders [31]. We believe that some cases of ADHD in 22q11DS preschoolers can be a nonspecific 'prodrome' presentation for variety of psychiatric disorders that will later 'crystalize' to other psychiatric diagnoses such as ASD and schizophrenia. This is supported by findings of a recent study showing that ADHD is a risk factor for the later development of psychotic disorders in 22q11DS [34].

\subsection{Comparison of psychiatric symptoms severity between 22q11DS and $i A S D$}

The children in our study with 22q11DS and ASD had similar scores on all CBCL scales besides withdrawal scores, on which children with iASD scored higher than the 22q11DS children (Table 3). These findings indicate that even though children with 22q11DS present with fewer impairments in the social-communication profile than children with ASD, they have the same severity of psychiatric symptomatology as children with iASD, emphasizing the disability of young children with 22q11DS.

\subsection{Comparison of the autism phenotype between 22q11DS and iASD}

To our knowledge, the current study is the second report that compared individuals with 22q11DS with individuals with idiopathic autism, and the first to compare the autism phenotype of 22q11DS to idiopathic autism in younger ages. As predicted, we found that children with 22q11DS diagnosed with ASD are less impaired than children with iASD in measures of ASD core symptoms, expressed by lower overall calibrated scores of the ADOS-2 $(p=0.007)$. In line with these findings, children with de novo mutations diagnosed with ASD showed less impairment in measures of ASD core symptoms in comparison to children with iASD [22].

Within the ADOS-2 subscales, we found less impairment in the social affect domain and similar severity in the restricted and repetitive behavior domain of the ADOS-2 in children with 22q11DS with autism compared to those with iASD. Within the social affect domain, nonverbal communication symptoms were less severe in our children with 22q11DS autism, in line with Kates et al. [11] who also observed that communication was less impaired in 22q11DS than in iASD and that repetitive behaviors were equally severe in both groups. The relatively high scores found in our 22q11DS group for the restricted and repetitive behavior domain is also in line with previous research led by members of the current study found that repetitive and restrictive behaviors, such as perseveration in speech and repetitive questions, were very common in 22q11DS, occurring in $53 \%$ of individuals with 22q11DS [35].

4.5. Comparison of the autism phenotype between 22q11DS with vs. without ASD

When comparing children with 22q11DS with ASD versus 22q11DS without ASD, we found that children with 22q11DS and
ASD had more severe ASD symptoms in all ADI-R and ADOS-2 domains besides the repetitive restricted behavior calibrated scores on the ADOS-2. As stated previously, high repetitive and restricted behaviors are common and debilitating in young children with 22q11DS [35], and as our study shows, this is common in 22q11DS both with and without autism. From our experience, when a child with 22q11DS is informed about exciting events (e.g, a gift he or she is expecting to receive) within a short notice rather than far ahead of time, it reduces his or her repetitive questions reflecting inability to delay gratification. Furthermore, when parents are guided how to better regulate the anxieties and phobias of their child with 22q11DS the repetitive questions related to future events they are anxious about are lessened.

\subsection{Limitations}

The main limitation of this study is the relatively small sample size, particularly the small number of children with 22q11DS and coexisting ASD. In addition, there were multiple comparisons conducted. Yet, most of the results of the analysis showed similar directionality and mostly in line with our hypotheses. The sample was composed mainly of males in order to match the children in the idiopathic autism group, thus precluding our ability to analyze potential sex effects. To overcome the small sample size, we chose to use non-parametrical analysis when comparing ASD symptomatology and psychiatric symptom severity between the 22q11DS subgroups and iASD group. It is noteworthy that because children with 22q11DS have a distinctive facial phenotype, we were not able to make this a blind study. The CBCL means in our study sample were relatively low- in the non-clinical range. Although the CBCL has been used in several studies of children with intellectual disabilities including in 22q11DS [7,8], to our knowledge there are no studies that provide norm-referenced data in youth with 22q11DS or other developmental disabilities. Possibly, the relatively lower than expected CBCL scores found in our study sample suggest that the thresholds for abnormal $\mathrm{CBCL}$ scale may be lower than those established for typically developing children.

The mean FSIQ in our cohort was higher than the typical FSIQ reported for 22q11DS [36]. It is assumed that FSIQ scores are somewhat higher in younger children than in older ones with 22q11DS, but there is limited empirical data on FSIQ in preschoolers with 22q11DS [36]. Most of the children with 22q11DS in our study were assessed with the short versions of the Wechsler test, which can lead to higher IQ estimates compared to full version assessments [37]. The fact that the iASD group was not available for the K-SADS evaluation, limited our ability to assess any differences in the prevalence of comorbid psychiatric disorders between the 22q11DS and iASD groups.

\section{Conclusion}

In conclusion, this is among the first studies to comprehensively assess the psychiatric phenotype in young children with 22q11DS. Using a rigorous clinical assessment, we found that $16 \%$ of young children with 22q11DS met DSM-5 criteria for ASD. We also found high rates of psychiatric disorders, specifically ADHD and anxiety, already apparent at a very young age. These findings stress the need for an early psychiatric assessment in this population. To our knowledge, there are overall limited data on the effectiveness of pharmacological and non-pharmacological treatments for individuals with 22q11DS, and none for preschoolers with 22q11DS. Because anxiety disorders and ADHD are risk factors for present and future maladjustment in 22q11DS, we believe study treatments targeting these disorders should be conducted with 22q11DS preschoolers. 


\section{Ethics}

The work described has been carried out in accordance with The Code of Ethics of the World Medical Association (Declaration of Helsinki) for experiments involving humans. Informed consent was obtained from all the subjects included in the study.

\section{Conflict of interest statement}

The authors declare no potential conflicts of interest with respect to the research, authorship and/or publication of this article.

\section{Funding}

This study was funded by the Binational Science Foundation, grant number 2017369; The National Institute of Mental Health of the National Institutes of Health under Award Number U01MH101722.

\section{Authors' contribution}

GD, SY and SC designed the study. SY, SFD, DK, SHD, WR and SC collected the original data. SY, GD and SFD managed and analyzed the data. SY, GD and SC wrote the first draft of the manuscript. All the authors contributed to and approved the final manuscript.

\section{Acknowledgments}

The authors thank all individuals and their parents who participated in the study.

\section{References}

[1] Michaelovsky E., Frisch A, Carmel M, Patya M, Zarchi O, Green T, et al. Genotype-phenotype correlation in 22q11. 2 deletion syndrome. BMC Med Genet 2012;13(December (1)):122.

[2] Bassett AS, McDonald-McGinn DM, Devriendt K, Digilio MC, Goldenberg P, Habel A, et al. International 22q11.2 Deletion Syndrome Consortium. Practical guidelines for managing patients with 22q11.2 deletion syndrome. J Pediatr 2011:159(August (2)) 332-9.e1.

[3] De Smedt B, Devriendt K, Fryns JP, Vogels A, Gewillig M, Swillen A. Intellectua abilities in a large sample of children with Velo-Cardio-Facial Syndrome: an update. J Intellect Disabil Res 2007;51(September Pt (9)):666-70.

[4] Swillen A, Vandeputte L, Cracco J, Maes B, Ghesquière P, Devriendt K, et al Neuropsychological, learning and psychosocial profile of primary school aged children with the velo-cardio-facial syndrome (22q11 deletion): evidence for a nonverbal learning disability? Child Neuropsychol 1999;5(December (4)):230-41.

[5] Schneider M, Debbané M, Bassett AS, Chow EW, Fung WL, van den Bree M, et al International consortium on brain and behavior in 22q11.2 deletion syndrome. Psychiatric disorders from childhood to adulthood in 22q11.2 deletion syndrome: results from the international consortium on brain and behavior in 22q11.2 deletion syndrome. Am J Psychiatry 2014;171(June (6)):627-39.

[6] Antshel KM, Aneja A, Strunge L, Peebles J, Fremont WP, Stallone K, et al. Autistic spectrum disorders in velo-cardio facial syndrome (22q11.2 deletion). J Autism Dev Disord 2007;37(October (9)):1776-86.

[7] Briegel W, Schneider M, Schwab KO. 22q11.2 deletion syndrome: behaviou problems of children and adolescents and parental stress. Child Care Health Dev 2008;34(November (6)):795-800.

[8] Klaassen P, Duijff S, Swanenburg de Veye H, Vorstman J, Beemer F, Sinnema G. Behavior in preschool children with the 22q11.2 deletion syndrome. Am J Med Genet A 2013;161A(January (1)):94-101.

[9] Angkustsiri K, Goodlin-Jones B, Deprey L, Brahmbhatt K, Harris S, Simon TJ. Social impairments in chromosome 22q11.2 deletion syndrome (22q11.2DS): autism spectrum disorder or a different endophenotype? J Autism Dev Disord 2014;44(April (4)):739-46.

[10] Fine SE, Weissman A, Gerdes M, Pinto-Martin J, Zackai EH, McDonald-McGinn DM, et al. Autism spectrum disorders and symptoms in children with molecularly confirmed 22q11.2 deletion syndrome. J Autism Dev Disord 2005;35(August (4)):461-70.

[11] Kates WR, Antshel KM, Fremont WP, Shprintzen RJ, Strunge LA, Burnette CP, et al. Comparing phenotypes in patients with idiopathic autism to patients with velocardiofacial syndrome (22q11 DS) with and without autism. Am J Med Genet A 2007;143A(November (22)):2642-50.

[12] Niklasson L, Rasmussen P, Oskarsdóttir S, Gillberg C. Neuropsychiatric disorders in the 22q11 deletion syndrome. Genet Med 2001;3(JanuaryFebruary (1)):79-84

[13] Niklasson L, Rasmussen P, Oskarsdóttir S, Gillberg C. Autism, ADHD, mental retardation and behavior problems in 100 individuals with 22q11 deletion syndrome. Res Dev Disabil 2009;30(July-August (4)):763-73.

[14] Ousley O, Evans AN, Fernandez-Carriba S, Smearman EL, Rockers K, Morrier MJ, et al. Examining the overlap between autism spectrum disorder and 22q11. 2 deletion syndrome. Int J Mol Sci 2017:18(May (5)):1071.

[15] Vorstman JA, Morcus ME, Duijff SN, Klaassen PW, Heineman-de Boer JA, Beemer FA, et al. The 22q11.2 deletion in children: high rate of autistic disorders and early onset of psychotic symptoms. J Am Acad Child Adolesc Psychiatry 2006;45(September (9)):1104-13.

[16] Achenbach TM, Rescorla L. ASEBA school-age forms \& profiles. Burlington (VT): University of Vermont, Research Center for Children, Youth, \& Families; 2001.

[17] Achenbach TM, Rescorla LA. Manual for the ASEBA preschool forms and profiles. Burlington (VT): University of Vermont, Research Center for Children, Youth, \& Families; 2000.

[18] Birmaher B, Ehmann M, Axelson DA, Goldstein BI, Monk K, Kalas C, et al. Schedule for affective disorders and schizophrenia for school-age children (KSADS-PL) for the assessment of preschool children-a preliminary psychometric study. J Psychiatr Res 2009;43(April (7)):680-6.

[19] Kim SH, Lord C. Combining information from multiple sources for the diagnosis of autism spectrum disorders for toddlers and young preschoolers from 12 to 47 months of age. J Child Psychol Psychiatry 2012;53(February (2)):143-51.

[20] Lord C, Rutter M, Le Couteur A. Autism diagnostic interview-revised: a revised version of a diagnostic interview for caregivers of individuals with possible pervasive developmental disorders. J Autism Dev Disord 1994;24(October (5)):659-85.

[21] Lord C, Rutter M, DiLavore PC, Risi S, Gotham K, Bishop S. Autism diagnostic observation schedule-Second edition (ADOS-2). Los Angeles: Western Psychological Services; 2012.

[22] Bishop SL, Farmer C, Bal V, Robinson EB, Willsey AJ, Werling DM, et al Identification of developmental and behavioral markers associated with genetic abnormalities in autism spectrum disorder. Am J Psychiatry 2017;174 (June (6)):576-85.

[23] Lord C, Risi S, Lambrecht L, Cook Jr. EH, Leventhal BL, DiLavore PC, et al. The autism diagnostic observation schedule-generic: a standard measure of social and communication deficits associated with the spectrum of autism. J Autism Dev Disord 2000;30(June (3)):205-23.

[24] Gotham K, Pickles A, Lord C. Standardizing ADOS scores for a measure of severity in autism spectrum disorders. J Autism Dev Disord 2009;39(May (5)):693-705.

[25] Hus V, Gotham K, Lord C. Standardizing ADOS domain scores: separating severity of social affect and restricted and repetitive behaviors. J Autism Dev Disord 2014;44(October (10)):2400-12.

[26] Silverstein AB. Full scale IQ equivalents for a two subtest short form of the wechsler preschool and primary scale of intelligence and the Wechsler intelligence scale for children revised. Psychol Rep 1983;53(1):16-8.

[27] Wechsler D. Preschool Scale of Intelligence ${ }^{\mathrm{TM}}$-third edition (WPPSI ${ }^{\mathrm{TM}}$-III). San Antonio. TX: Harcourt Assessment; 2002.

[28] Wechsler D. Wechsler intelligence scale for children-WISC-IV. Psychological Corporation; 2003.

[29] Mullen EM. Infant MSEL manual: infant mullen scales of early learning. American Guidance Service; 1989.

[30] Swineford LB, Guthrie W, Thurm A. Convergent and divergent validity of the Mullen scales of early learning in young children with and without autism spectrum disorder. Psychol Assess 2015;27(December (4)):1364-78.

[31] Law EC, Sideridis GD, Prock LA, Sheridan MA. Attention-deficit/hyperactivity disorder in young children: predictors of diagnostic stability. Pediatrics 2014;133(April (4)):659-67.

[32] Daniels AM, Mandell DS. Explaining differences in age at autism spectrum disorder diagnosis: a critical review. Autism 2014;18(July (5)):583-97.

[33] ZERO TO THREE. DC:0-5 $5^{\mathrm{TM}}$ : diagnostic classification of mental health and developmental disorders of infancy and early childhood Washington, DC. 2016.

[34] Niarchou M, Chawner SJ, Fiksinski A, Vorstman JA, Maeder J, Schneider M, et al. Attention deficit hyperactivity disorder symptoms as antecedents of later psychotic outcomes in 22q11. 2 deletion syndrome. Schizophrenia research. 2018 August 7.

[35] Gothelf D, Presburger G, Zohar AH, Burg M, Nahmani A, Frydman M, et al. Obsessive-compulsive disorder in patients with velocardiofacial (22q11 deletion) syndrome. Am J Med Genet B Neuropsychiatr Genet 2004;126B(April (1)):99-105.

[36] Swillen A. The importance of understanding cognitive trajectories: the case of 22q11.2 deletion syndrome. Curr Opin Psychiatry 2016;29(March (2)):133-7.

[37] Hurks P, Hendriksen J, Dek J, Kooij A. Accuracy of short forms of the Dutch Wechsler preschool and primary scale of intelligence: third edition. Assessment 2016;23(April (2)):240-9. 